\title{
Contents, Vol. 12, 1904
}

\section{Inhalts -Verzeichnis.}

Orig $\cdot$ inalarbeiten.

Bach, L., Das Verhalten der Pupillen bei der Konvergenz und Akkommodation 725

Bartels, Martin, Pupillenverhältnisse bei Neugeborenen . 638

Bielschowsky, A., Ueber die Genese einseitiger Yertikal-

bewegungen der Augen 545

Ewetzky, ïh. von und J. von Kennel, Eine Fliegenlarve in der vorderen Augenkammer. (Hierzu Taf. III.) . 337

Gleichen, A., Neue Theorie der Schattenprobe (Skiaskopie) 1

- Noch einmal meine Skiaskopie-Theorie 653

Grönholm, V., Ueber die Verbreitung des Trachoms und der Blindheit in Filmland. (Hierzu Taf. IY u. Y.) . 425

Grunert, Karl, Erfahrungen aus dem Gebiete der Chirurgieder oberen Nebenhöhlen der Nase, mit besondererBerücksichtigung der postoperativen Augenmuskel-störungen 762

Hamburger, G, Die anatomischen und ptiysiologischen Grundlagen der Prof. Stillingsçhen Theorie über Ent-stehung und Bedeutung der Kurzsichtigkeit .... 351

Herzog, II., Pathologie der Cilien. II. Abschnitt. . . 180

J urnitschek, Felix, Em Fall von ïarsitis syphilitica . . 376

Kennel, J. von, s. Ewetzky u. K.

Krauss, $\Lambda$ Vilhelm, Zur intraokularen Desinfektion, mit besonderer Berücksichtigung des

Jodoforms. (HierzuTaf. II.) 97

Kuhnt, H., Ueber Ausziehung des einfachen Altersstars 456

Loeser, Ueber das kombinierte Yorkommen von Myasthenie und Baseclowscher Krankheit, nebst Bemerkungen über die okulären Symptome der Myasthenie . . . 368

Matys, W., Ein Fall von Parinauds Conjunctivitis. (Hierzu

Taf. YI.) $\quad .557$

May, Bruno, Keratitis punctata und Glaukom . · · 309

$-\mathrm{IV}-$

Munch, Karl, Ueber die muskulöse Natur des Stromazell-

netzes der Uvea 525

Nacht, Albert, Die bei Erkrankungen der Meningen be-obacliteten Erscheinungen, mit spezieller Berück-

siclitigung der okularen Symptome 243

Onodi, A., Die Sehstörungen und Erblindung nasalen Ur-sprunges, bedingt durch Erkrankungen der hinteren

Nebenhöhlen 23

Perlmann, Alfred, Zur Sideroskopie 651 
Pes, 0., Ueber die akute gangränöse Phlegmone der Lider 438 Peters, A., Zur Frage der Sehstörungen durch Autosuggestion 17 Pfalz, G., Die Spätdiagnose traumatischei

Netzliautablösung 386 Pilcz, Alexander, und Hugo Wintersteiner, Ueber Er-gebnisse von Augenspiegeluntersuchungen an Geistes-kranken, mit besonderer Berücksichtigung der kongenitalen Anomalien $\quad 729$

Schanz, Fritz, Eine neue Visiervorrichtung, welche auchbei herabgesetzter Beleuchtung und in der Nacht ver-wendbar ist $\quad 572$

- $\quad$ Ueber das Sehenlernen blindgeborener und später mit

Erfolg operierter Menschen 753

Tertsch, Rudolf, Ein Beitrag zu den dem Diphtherie-

bazillus ähnlichen Stäbchen des Conjunktivalsackes . 621Wintersteiner, Hugo, s. Pilcz u.

W.Wolff, Hugo, Ueber die Skiaskopie-Theorie 490

- Ueber die Abnahme der Pupillarreflexempfindlichkeit der

Netzhaut vom Zentrum nach der Peripherie .... 644Zia, H., Ueber ausgedehnte Knochen- und

Markbildung imAuge, sowie über auffällige fibrinähnliche Gebilde inder verkalkten Linse.

(Hierzu Taf. I.)

47

Berichte über die deutsehe ophthalmolog $\cdot$ ische Literatur.

Diagnostik und Extraktion von Fremdkörpern (1903). Von

Dr. Ed. Asmus in Düsseldorf 53

Pathologische Anatomie (1903). Von Prof. Dr. von Michel

in Berlin 68, 393

Anatomie des Auges (II. Semester 1903). Von Prof. Dr.

Sobotta in Würzburg 589

Dioptrik und Anomalien der Refraktion und Akkommodation(I. Semester 1904). Von Doz. Dr.

W. Salzmannin Wien 657

$\mathrm{y}_{-}$

Bepiehte übep die auslandisehe ophthalmologisehe Litepatup.

Die amerikanische Literatar. Von Dr. Denig in New-York.

(I. Semester 1903) 495

Die englische Literatar. Von Dr. Grruber in London.

(II. Semester 1903) ' .... 502

Die ungariscke Literatur. Von Prof. Dr. E. v. Grósz in

Budapest. (I. and II. Semester 1903) 782

Gesellsehaftsbepiehte.

Arb itspi"ogratnm des X. Internation alen Opktkal-

mologenkongresses in Luzern515

Berickt über den X. Internationalen Opktkalmo-1 ogenkongress ínLuzern. 13. - 17.Se ptember 1904.Von Dr. B. Pollack in Berlin 603, 680

76. Versammlung deutsclier $\mathrm{N}$ aturf orsc lier und A erzte in Breslau. Abteilung Augenlieilkunde.

Von Dr. Paul und Dr. Wernicke in Breslau .... 661

Berliner ophthalmologisclie Ge sell sc h aft.

Sitzung vom 16. J uni 190486

Sitzung vom 20. Oktober 1904 . 792

Wiener ophtlialmologische Gesellscliaf t.

Sitzung vom 13. April 1904409

Sitzung vom 19. Oktober $1904 \quad 795$ 
St. Peters burger Ophthalmologisclie G-e sells chaft.

Sitzung vom 22. Januar 1904797

Sitzung vom 19. Februar 1901. 798

Sitzung vom 22. April 1904799

Sitzung vom 6. Mai 1904 . 799

"6

Unfall- und Vepsiehepung ·skunde.

Unfall- und Versicherungskunde 412, 512

Thepapeutische ümsehau.

Therapeutische Umschau ... 88, 416, 514, 616, 718, 800

Offene Koppespondenz.

Offene Korrespondenz

87

Tag esnaehpiehten und Notizen.

Tagesnachrichten und Notizen ..... 92, 420, 618, 720

Buchanzeig $\cdot e$.

Landolt, E., Die Untersuchungsmethoden 90

L. Litepatup - Vepzeichnis.

Literatur-Verzeichnis .... 93

421

521

618

721

802 